\title{
Luc Racine
}

Sociologue, Département de sociologie, Université de Montréal

(1985)

\section{"Du mythe à l'utopie: éclaircissements typologiques et analyse d'un cas"}

Un document produit en version numérique par Jean-Marie Tremblay, bénévole, professeur de sociologie au Cégep de Chicoutimi

Courriel: jean-marie tremblay@uqac.ca

Site web pédagogique : http://www.uqac.ca/jmt-sociologue/

Dans le cadre de: "Les classiques des sciences sociales" Une bibliothèque numérique fondée et dirigée par Jean-Marie Tremblay, professeur de sociologie au Cégep de Chicoutimi Site web: http://classiques.uqac.ca/

Une collection développée en collaboration avec la Bibliothèque Paul-Émile-Boulet de l'Université du Québec à Chicoutimi Site web: http://bibliotheque.uqac.ca/ 


\section{Politique d'utilisation de la bibliothèque des Classiques}

Toute reproduction et rediffusion de nos fichiers est interdite, même avec la mention de leur provenance, sans l'autorisation formelle, écrite, du fondateur des Classiques des sciences sociales, Jean-Marie Tremblay, sociologue.

Les fichiers des Classiques des sciences sociales ne peuvent sans autorisation formelle:

- être hébergés (en fichier ou page web, en totalité ou en partie) sur un serveur autre que celui des Classiques.

- servir de base de travail à un autre fichier modifié ensuite par tout autre moyen (couleur, police, mise en page, extraits, support, etc...),

Les fichiers (.html, .doc, .pdf., .rtf, .jpg, .gif) disponibles sur le site Les Classiques des sciences sociales sont la propriété des Classiques des sciences sociales, un organisme à but non lucratif composé exclusivement de bénévoles.

Ils sont disponibles pour une utilisation intellectuelle et personnelle et, en aucun cas, commerciale. Toute utilisation à des fins commerciales des fichiers sur ce site est strictement interdite et toute rediffusion est également strictement interdite.

L'accès à notre travail est libre et gratuit à tous les utilisateurs. C'est notre mission.

Jean-Marie Tremblay, sociologue

Fondateur et Président-directeur général, LES CLASSIQUES DES SCIENCES SOCIALES. 
Cette édition électronique a été réalisée par Jean-Marie Tremblay, bénévole, professeur de sociologie au Cégep de Chicoutimi à partir de :

Luc Racine

Sociologue, Département de sociologie, Université de Montréal

“Du mythe à l'utopie : éclaircissements typologiques et analyse d'un cas”.

Un article publié dans la revue Anthropologie et Sociétés, vol. 9, no 1, 1985, pp. 13-31. Numéro intitulé : “Utopies”. Québec : Département d'anthropologie, Université Laval.

M Luc Racine, sociologue, professeur au département de sociologie de l’Université de Montréal, nous a accordé le son autorisation de diffuser électroniquement cet article dans Les Classiques des sciences sociales.

Courriel : luc.racine@umontreal.ca

Polices de caractères utilisée :

Pour le texte: Times New Roman, 14 points.

Pour les citations : Times New Roman, 12 points.

Pour les notes de bas de page : Times New Roman, 12 points.

Édition électronique réalisée avec le traitement de textes Microsoft Word 2004 pour Macintosh.

Mise en page sur papier format : LETTRE (US letter), 8.5’’ x 11'’)

Édition numérique réalisée le 10 juillet 2007 à Chicoutimi, Ville de Saguenay, province de Québec, Canada. 


\section{Luc Racine}

Sociologue, Département de sociologie, Université de Montréal

\section{"Du mythe à l'utopie: \\ éclaircissements typologiques et analyse d'un cas”}

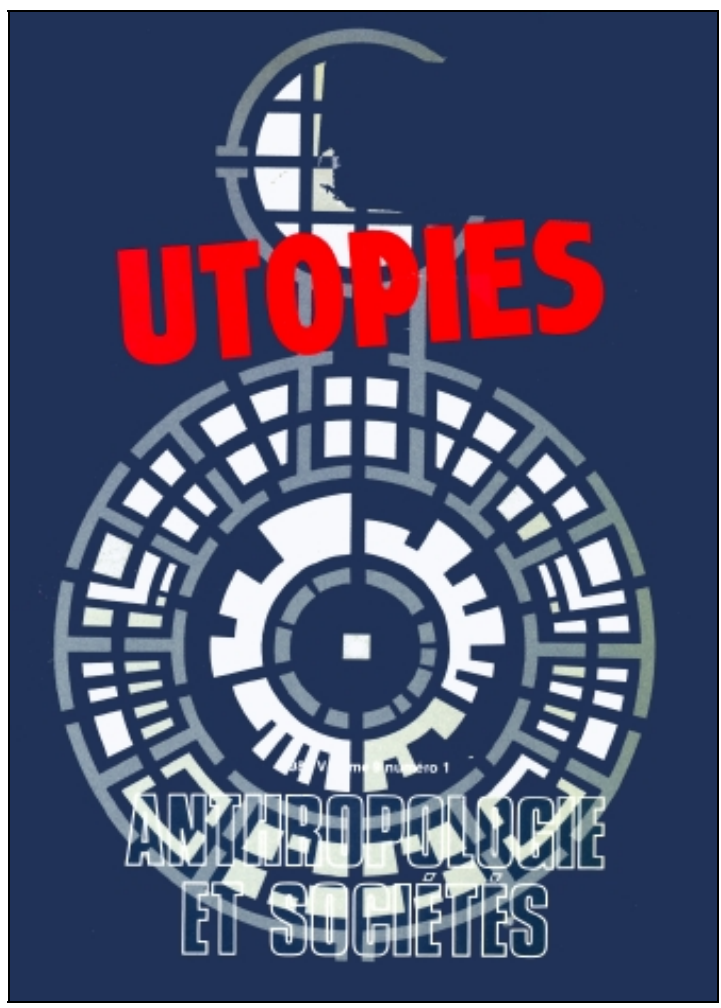

Un article publié dans la revue Anthropologie et Sociétés, vol. 9, no 1, 1985, pp. 13-31. Numéro intitulé : “Utopies”. Québec : Département d'anthropologie, Université Laval. 


\section{Table des matières}

$\underline{\text { Introduction }}$

Les différentes formes de l'état idéal de perfection sociale Analyse du Fast

Les traits constants de l'état idéal de perfection sociale

Libération des diverses formes du mal

La parfaite communication avec autrui

$\underline{\text { L'harmonie des rapports sociaux }}$

Les caractéristiques utopiques

Les moyens de réalisation de l'état idéal

La conception de l'espace

La conception du temps

Pour conclure

Appendice : Résumé de la nouvelle de Fast

Bibliographie 
Luc Racine

Sociologue, Département de sociologie, Université de Montréal

“Du mythe à l'utopie : éclaircissements typologiques et analyse d'un cas”.

Un article publié dans la revue Anthropologie et Sociétés, vol. 9, no 1, 1985, pp. 13-31. Numéro intitulé : “Utopies”. Québec : Département d'anthropologie, Université Laval.

\section{Introduction}

$\underline{\text { Retour à la table des matières }}$

L'analyse que nous présentons ici est une tentative d'illustration d'une typologie que nous avons déjà présentée en détail ailleurs. Ce travail proposait une classification des principales formes que peut revêtir, selon l'imaginaire de divers types de sociétés, l'état idéal de perfection des rapports sociaux: mythe paradisiaque, âge d'or, royaume millénaire et cité utopique (Racine, 1983). Nous résumerons d'abord ici l'essentiel de ce travail : traits communs à toutes les formes d'état idéal de perfection sociale, pôles extrêmes de la typologie (mythe paradisiaque et utopie), critères permettant de distinguer les unes des autres les diverses formes en question. Puis le plus important de notre propos sera de montrer comment la typologie permet d'analyser un matériel concret avec précision.

Nous analyserons un seul cas. Une typologie est un outil descriptif dont l'utilité ne consiste que dans sa plus ou moins grande capacité à classer et à décrire sans ambiguïté les objets différents relevant d'un même domaine. Elle doit donc être assez précise en ses critères pour permettre l'analyse de cas concrets, et il nous a semblé préférable d'approfondir un seul exemple plutôt que de multiplier des illustrations rapides et forcément superficielles. Il ne doit donc pas y avoir de 
malentendu sur la portée de notre analyse : elle ne valide pas la typologie, elle indique la démarche générale qu'il est bon d'utiliser si l'on veut s'en servir.

D'autre part, si nous n'avons pas choisi d'examiner un texte utopique classique, c'est avant tout pour des raisons techniques. L'analyse d'un texte de quelques centaines de pages est extrêmement lourde à présenter, et il est alors tentant d'omettre tous les détails qui permettraient justement de saisir la méthode en action. C'est ce qui nous a poussé à choisir une nouvelle d'environ soixante-dix pages, écrite par un romancier américain contemporain. Sans être une figure dominante de la littérature, Howard Fast a connu une certaine notoriété pour la réalisation cinématographique de plusieurs de ses romans, tels Spartacus et La passion de Sacco et Vanzetti. La nouvelle

que nous analyserons est intitulée "Les premiers hommes » et, comme l'ensemble des nouvelles constitué par le recueil d'où elle est tirée (Au seuil du futur, Gérard \& Cie, Verviers, 1962), elle représente une des incursions de son auteur dans le champ de la littérature d'anticipation. Remarquablement bien construite, très dense, elle facilitait particulièrement notre propos.

\section{Les différentes formes de l'état idéal de perfection sociale 1}

$\underline{\text { Retour à la table des matières }}$

Pour qui s'intéresse aux diverses façons qu'ont eues les sociétés humaines d'imaginer un état idéal de perfection ou d'harmonie sociale, il s'avère crucial de ne pas confondre, comme c'est trop souvent le cas, des systèmes de représentations aussi différents que la pensée mythi-

1 Pour plus de détails et de références sur la typologie que nous résumons ici, cf. Racine (1983). 
que, le millénarisme et l'utopie ${ }^{2}$. Le paradis primitif, l'âge d'or et le royaume millénaire ne sont pas de simples précurseurs de la cité idéale des utopistes. L'utopie n'est qu'un système de représentation de l'état de perfection sociale parmi d'autres : le plus récent. Entre le paradis primitif et la cité utopique, ce sont aussi bien les variations que les points communs qu'il importe de mettre en évidence. Et ce qui varie, ce ne sont pas tant certains contenus de l'état idéal que sa localisation spatio-temporelle et les moyens à mettre en oeuvre pour y accéder. Un examen attentif des diverses descriptions de l'âge d'or et du paradis, du royaume millénaire et de la cité utopique, révèle en effet l'existence d'un nombre surprenant de traits communs à ces diverses représentations de l'état idéal. Nous nous bornerons ici à souligner les plus marquants ${ }^{3}:$ a) absence de travail pénible, de souffrance physique et morale, longévité ou immortalité ; b) communication parfaite avec autrui (autres humains, animaux, êtres divins), qui implique souvent nudité et liberté sexuelle ${ }^{4}$; c) fonctionnement harmonieux des rapports sociaux, qui n'entraîne pas nécessairement un fonctionnement égalitaire : chacun est satisfait de remplir son rôle, qu'il y ait hiérarchie ou égalitarisme, spontanéité ou organisation planifiée ${ }^{5}$.

2 Une telle confusion se trouve, entre autres, chez Duveau (1961), Manuel et Manuel (1979), Manheim (1956), Servier (1967).

3 Sur la présence constante de ces traits, cf. Eliade (1949 : 139-205) ; Eliade (1962 : 181-232) ; Eliade (1957 : 40-59 et 78-94). Sur les diverses représentations du paradis, cf. Guhl (1972) pour une revue d'ensemble ; Corbin (1953, 1963) pour la tradition iranienne et Daniélou (1953) pour le christianisme. Sur le royaume millénaire, cf. Barret/Gurgand (1981), Burridge (1969), Clastres (1975), Cohn (1967, 1910), Desroches (1969), Hobsbawm (1963), Lanternari (1962), Queiroz (1968), Rigaux (1932), Servier (1967), Thrupp (1962), Vulliaud (1952), Worsley (1957). Sur l'utopie, cf. Debout (1978), Desanti (1970), Desroches (1972, 1975), Eurich (1967), Ferguson (1975), Lapouge (1978), Manuel et Manuel (1979), Mucchielli (1960), Mumford (1966), Ruyer (1950), Suvin (1977), Versins (1972), Wunenburger (1979).

4 Sur la nudité et la liberté sexuelle comme traits constants de l'imaginaire d'un état social idéal, cf. Eliade (1962 : 181-232) et Desroches (1978).

5 Il faut Prendre garde de ne pas transposer l'idéologie égalitaire à d'autres types de sociétés que la nôtre (sur l'idéologie égalitaire et la hiérarchie, cf. les travaux de L. Dumont $(1966,1977,1983)$. Les mythes du paradis et de l'âge d'or parlent de justice et d'abondance plutôt que d'égalité : ... /... cf. par exemple, 
La réalisation de chacun de ces traits varie évidemment d'un type à l'autre : l'absence de maladie peut être assurée aussi bien par les progrès de la médecine moderne que par la protection des dieux, l'absence de travail tout autant par des pratiques magiques que par l'automation, l'éternelle jeunesse aussi bien par l'élixir de vie que par la biologie, la communication parfaite tout autant par la télépathie que par la « révolution » télématique...

Mais les différences importantes ne se situent pas principalement à ce niveau. Ce qui différencie la pensée mythique et la pensée utopique en ce qui concerne l'état social idéal, ce sont la localisation spatiotemporelle de cet état et les moyens mis en oeuvre pour y accéder ${ }^{6}$. Pour ce qui est de la pensée mythique, les choses se présentent schématiquement de la manière suivante. $A u$ temps de l'origine et de la création, a prévalu pour l'humanité un état de bonheur, d'harmonie et de perfection, état ensuite aboli à la suite d'une faute rituelle quelconque. Depuis lors dominent les conditions de vie coutumières à l'humanité actuelle : mort et sexualité, travail et souffrance, destruction et dégénérescence dues au passage du temps. Périodiquement, rites et cérémonies viennent abolir symboliquement ces effets dévastateurs du temps. Fêtes et orgies, quand s'abolissent ou s'inversent les règles sociales, témoignent d'un retour au chaos qui prélude à toute nouvelle création et permettent la réactualisation du temps paradisia-

Hésiode, Les travaux et les jours (90-110) et Virgile, IVe Bucolique ; pour l'Inde, cf. Eliade (1952 : 73-119). Dans les millénarismes et messianismes, il n'y a presque jamais égalité politique entre le leader, ses acolytes et les fidèles, pas toujours égalité économique réelle, et rarement égalité entre hommes et femmes. Quant aux utopies, il serait difficile de dire égalitaire la République de Platon ou l'Abbaye de Thélème de Rabelais ; l'égalitarisme de More est essentiellement économique, il n'y a sur son [le ni égalité politique ni égalité entre les sexes.

6 Sur ces critères de différenciation, cf. Eliade (1963, 1969), Desroches (1969, 1973) et Wunenburger (1979). 
que des origines. Par ces rites et cérémonies, il est possible de réintégrer collectivement la condition paradisiaque ${ }^{7}$.

La pensée mythique met ainsi en jeu une conception cyclique du temps, le passage de celui-ci étant négativement valorisé. L'état de perfection sociale ne se situe pas dans un espace accessible durablement avant la mort et il réside temporellement en un passé immémorial qui se confond paradoxalement avec l'éternel présent. Jamais le paradis n'est oeuvre humaine, et toujours un don des dieux : le rôle de l'humanité se borne ici à réactualiser rituellement la destruction et la reconstruction symbolique du cosmos ${ }^{8}$.

C'est aux antipodes de tout cela que se situe la pensée utopique, qui conçoit l'état de perfection sociale comme réalisable par l'homme en ce monde, de façon durable, dans un avenir plus ou moins rapproché, et ce par le moyen de la science, de la technique, de la rationalisation des rapports sociaux ${ }^{9}$. Le temps est ici linéaire, irréversible et positivement valorisé, il coule vers un avenir conçu comme seul lieu de la libération humaine, sans retour possible vers un passé que valorise toutefois partiellement son rôle d'ébauche du présent et de l'avenir ${ }^{10}$.

Le tableau suivant regroupe schématiquement ces diverses oppositions 11 . Il s'agit bien entendu de pôles extrêmes, correspondant, à la limite, aux mythes paradisiaques des sociétés de chasseurs-cueilleurs

7 Dans ce paragraphe, je résume l'essentiel de la présentation d'Eliade 0 963, 1969). Sur la conception cyclique du temps, on verra aussi Caillois (1967), et Paz (1976).

8 Cf. Eliade (1949), chap. VII-XII.

9 Sur le lien existant entre science, rationalisme et utopie, cf. Desroches (1972), Eurich (1967), Suvin (1977), Wunenberger (1979).

10 Pour une analyse des diverses variantes et de l'évolution de la pensée utopique, cf. les références de la note 3), et Racine (1983). En ce qui concerne la fascination pour les origines dans la pensée occidentale, cf. Boas (1966) et Schlanger (1971).

11 Comparer ce tableau aux schémas donnés par Desroches (1973) et par Wunenburger (1979). 
et aux utopies libérales ou socialistes des sociétés industrielles. La théorie des âges cycliques de l'humanité et les divers millénarismes, dont nous ne nous occuperons pas ici, présentent une série d'intermédiaires entre ces pôles ${ }^{12}$.

\section{Principales oppositions entre mythe paradisiaque et utopie}

$\underline{\text { Retour à la table des matières }}$

\begin{tabular}{|l|l|l|l|l|l|l|}
\hline & \multicolumn{2}{|c|}{ Temps } & Espace & \multicolumn{2}{c|}{ Accès } \\
\hline & $\begin{array}{l}\text { Localisa- } \\
\text { tion de } \\
\text { l'état } \\
\text { idéal }\end{array}$ & Direction & Valeur & & Modes & Agents \\
\hline Mythe & passé & cyclique & négative & $\begin{array}{l}\text { inacces- } \\
\text { sible en } \\
\text { ce monde } \\
\text { de ma- } \\
\text { nière } \\
\text { durable }\end{array}$ & $\begin{array}{l}\text { qumboli- } \\
\text { que (fê- } \\
\text { tes, rites, } \\
\text { etc.) }\end{array}$ & dieux \\
\hline Utopie & avenir & linéaire & positive & $\begin{array}{l}\text { accessi- } \\
\text { ble en ce } \\
\text { monde } \\
\text { de ma- } \\
\text { nière } \\
\text { perma- } \\
\text { nente }\end{array}$ & $\begin{array}{l}\text { (science, } \\
\text { politi- } \\
\text { que) }\end{array}$ & humains \\
\hline
\end{tabular}

1212 Plus de détails dans Racine (1983). 


\section{ANALYSE DU RÉCIT DE FAST}

$\underline{\text { Retour à la table des matières }}$

On trouvera en appendice un résumé de la nouvelle de Fast. L'analyse que nous présentons ici se déroulera en deux grandes étapes. Nous montrerons d'abord comment les traits constants d'un état idéal de perfection sociale se retrouvent tous d'une manière ou d'une autre, dans ce texte : a) libération des diverses formes du mal et de la souffrance (travail pénible, mort et solitude, maladie, haine) ; b) parfaite communication avec autrui (redéfinition du rapport entre soi et l'autre, libération de la sexualité) ; c) harmonie des rapports sociaux. Puis, en une seconde étape, nous dégagerons les Caractéristiques qui permettent de classer le récit au sein de l'imaginaire utopique : accessibilité de l'état idéal en ce monde, par le moyen de la science, conception linéaire d'un temps positivement valorisé.

\section{Les traits constants de l'état idéal de perfection sociale}

Comme nous allons le constater, rien ne manque ici des traits caractérisant tout état social idéal : le mal disparaît, la communication est parfaite, l'harmonie est totale. Mais C'est d'un processus qu'il s'agit, cet état n'est pas atteint d'un coup : le récit de Fast n'a pas l'allure de description statique propre à la littérature utopique classique.

\section{Libération des diverses formes du mal}

Tout au long du récit, l'apparition des pouvoirs psychiques chez les enfants (télépathie et psychokinésie) est étroitement liée à la disparition rapide du travail pénible, de la mort et de la solitude, de la maladie, de la souffrance et de la haine. 


\section{Le travail}

Le développement intellectuel foudroyant des enfants, auquel vient vite se joindre l'apparition de pouvoirs insoupçonnés de la pensée sur la matière (psychokinésie), libère l'ensemble des membres de la réserve de tout travail pénible. À six ans, un petit Bantou et une petite Italienne construisent un appareil à mesurer la lumière (p. 55) ${ }^{13}$. Les maîtres adultes sont vite dépassés 14 : leurs enfants dissolvent un roc par la seule puissance de la pensée, ils construisent une mini-centrale atomique pour alimenter la réserve en énergie, ils s'équipent de véhicules propulsés par des champs d'énergie inconnue (pp. 72-73). Capables de pénétrer mentalement les atomes et de réarranger les électrons, de tirer un élément d'un autre, leur contrôle sur la matière est sans aucune mesure avec ce que connaît la technologie ordinaire 15.

\section{La mort et la solitude}

Dès que les adultes constatent l'apparition de la capacité télépathique au sein du groupe d'enfants, c'est le professeur Goldbaum qui en dégage les implications radicales.

Si un de ces enfants peut réellement lire dans l'esprit des autres, ils n'auront plus qu'une seule mémoire, qui sera leur mémoire à tous. Ils partageront toutes leurs expériences, toutes leurs connaissances, tous leurs rêves et ils deviendront immortels. Car, lorsque l'un d'eux mourra, un autre sera

13 Toute indication de pagination de ce genre renvoie à Fast (1962).

14 Un physicien célèbre, Alex Cromwell, commence à apprendre la physique aux enfants : « Huit ans plus tard, les enfants apprenaient la physique à Cromwell » (P. 72). Un an plus tard : " Ils voulaient expliquer à Cromwell comment leurs seules pensées pouvaient atteindre un tel résultat (dissoudre un rocher), mais, pas plus que nous, le physicien n'était à même de les suivre » (p. 73).

15 «La puissance mentale des enfants leur permet de pénétrer les atomes, de réarranger les électrons, de tirer un élément d'un autre - et ils considèrent cela comme l'enfance de l'art, comme des tours de prestidigitation tout juste bons à nous amuser et à nous ébahir » (p. 74). 
admis dans l'ensemble, puis un autre et un autre encore. La mort perdra toute sa signification (pp. 64-65).

Ainsi, la personnalité de chacun survivra à la perte du corps, au sein du groupe télépathique, il n'y aura plus ni mort ni solitude. C'est pourquoi les enfants n'éprouvent pas de peine lorsque J'un d'eux meurt, contrairement à ce qui se passe lors de la mort de l'un des adultes, qui est irréversible et sans appel 16.

\section{La maladie et la souffrance}

Peu après leur neuvième année, télépathie et psychokinésie combinées permettent aux enfants d'en finir avec les souffrances physiques et morales. Si l'un d'entre eux tombe malade, ils n'ont « qu'à unir leurs pensées pour s'identifier au malade et le guérir » (p. 69). Ce pouvoir, ils l'emploient aussi pour garder les adultes en aussi bonne santé que possible, pour dissiper leurs amertumes et calmer leurs craintes ${ }^{17}$. Ils l'appliquent également aux animaux, endormant les chats et les chiens vieux et malades d'un sommeil paisible et définitif, procédant de même pour tuer les animaux de boucherie (p, 78).

\section{La haine et la méchanceté}

Les enfants deviennent très vite absolument réfractaires à toutes les formes de méchanceté : dans leurs jeux, ils cessent très tôt de se mettre en colère et de se moquer (p. 69). Même pour protéger la réserve

16 « Les enfants ne Purent jamais vraiment comprendre comment nous pouvions supporter notre solitude, comment nous pouvions vivre en sachant que la mort sera pour nous l'extinction la plus totale (...). Pour eux, la mort est une chose totalement différente : la perte du corps, sans plus ; la personnalité elle-même est immortelle puisqu'elle survit chez les autres, de façon consciente » (p. 68).

17 Vers la fin du récit, l'un des adolescents s'adresse aux adultes en ces termes : " Peut-être avons-nous un peu contribué à dissiper vos amertumes, à vous garder en aussi bonne santé que possible et à calmer ce fouillis de craintes et de cauchemars que vous appelez sommeil » (p. 75). 
d'une intrusion, ils se refuseront plus tard, devenus adolescents, à toute violence et à tout affrontement :

Ils sont incapables de faire souffrir un être humain et à plus forte raison de le tuer (...). Ils ne peuvent pas tuer, ils ne peuvent pas faire du mal (pp. 7879) 18 .

\section{La parfaite communication avec autrui}

Le développement de la télépathie et la libération de la sexualité redéfinissent complètement les rapports entre soi et autrui, alors que s'établit entre les enfants une communauté de type fusionnel.

18 Presqu'à l'opposé de ce que nous venons de décrire, l'ancienne humanité se caractérise par l'hégémonie de la solitude, de la mort, de la haine, et par la limitation des capacités humaines.

a) Emprise de la haine. Lors d'une discussion concernant l'apparition de la télépathie chez les enfants, le professeur Goldbaum résume bien la situation : « Nous tuons, nous torturons, nous exaltons le meurtre et la fausseté et l'hypocrisie et la superstition ; nous détruisons notre propre corps par des drogues et une nourriture empoisonnée; nous trompons notre prochain et nous nous trompons nous-mêmes -et nous ne respirons que la haine, toujours la haine » (p. 64). Rempli de terreur, l'ancien monde fait tout pour empêcher l'apparition d'une humanité nouvelle : "Dans le passé, qu'est-il arrivé aux gens qui entendaient des voix, que l'on disait possédés du démon ? Quelques-uns sont devenus des saints, mais combien ont été brûlés sur l'échafaud ?» (p. 77).

b) Limitations des possibilités humaines. D'après la théorie des Arbalaid, la société humaine a toujours étouffé dès le plus jeune âge les potentialités d'un véritable développement de l'humanité : «Si l'homme-plus existe, il est pris au piège et encagé dans son environnement humain, aussi sûrement qu'un enfant élevé par des animaux l'est par ces animaux » (p. 27).

c) La solitude et la mort. Le professeur Goldbaum : « Nous avons vécu dans les ténèbres, chacun de nous affrontant l'existence avec son pauvre cerveau pour mourir en emportant les souvenirs d'une vie. Nous ne devons pas nous étonner d'avoir accompli aussi peu de choses » (p. 65). 


\section{La libération de la sexualité}

C'est le retour à la nudité, au naturel, à l'innocence. Les enfants sont en ce domaine semblables à des païens ou à des dieux, ils ne connaissent ni le mai ni l'excès, ils s'aiment avec « une grâce infinie et une joie profonde » :

La plupart du temps, ils ne portent aucun vêtement. Pour eux, la sexualité a toujours été une joie, jamais un problème (...). Je vis avec des garçons et des filles qui ne connaissent pas le mal, semblables à des dieux ou à des païens (pp. 56-57).

La description d'une sexualité joyeuse et innocente, faite de grâce et de naturel, évoque les images du paradis d'avant la chute, l'état édénique où le mal n'était pas encore apparu sur terre. Quant aux rapports de couple, on sait qu'ils sont maintenus entre les adultes, bien que la prise en charge des enfants soit collective (chaque adulte étant père ou mère de tous). Pour ce qui est du groupe d'enfants, toutefois, ce rapport est pour le moins complètement déprivatisé. Grâce à la télépathie, chacun est à la fois lui-même et tous les autres, possédant l'expérience d'une femme aussi bien que celle d'un homme. On peut difficilement imaginer qu'existent dans un tel groupe des relations exclusives :

Les garçons et les filles font l'amour sans aucune inhibition et leur plaisir est partagé par tous (p. 68).

\section{La communauté fusionnelle et le rapport à autrui}

Puisque chacun participe directement à la totalité de l'expérience et de la mémoire des autres, il ne saurait y avoir « ni mensonge, ni tromperie, ni rationalisation, ni secret, ni sentiment de culpabilité » (p. 63). $\mathrm{Au}$ point de vue éthique, un tel état fusionnel implique que personne 
ne fera à autrui ce qu'il ne se ferait pas à lui-même ${ }^{19}$. Cela crée sur le plan affectif, entre les enfants, un amour d'une intensité encore plus profonde que celle de l'amour maternel 20, et entraîne une extrême solidarité, un soutien mutuel constant ${ }^{21}$. Il n'y a plus ni solitude, ni incommunicabilité entre les sexes :

Chacun est présent dans le corps de ses trente-neuf camarades, chacun possède sa propre personnalité et celles de tous les autres - et elles sont parties intégrantes de lui-même. Chacun se retrouvant dans tous les autres vit à la fois la vie d'un homme et la vie d'une femme (pp. 66-67).

Dans le domaine intellectuel, enfin, le groupe se comporte comme un esprit unique, « l'esprit de quarante enfants prodigieux » (p. 71). La télépathie assure un fonctionnement symbolique infiniment supérieur au langage articulé, et les enfants se créent ainsi un langage « aussi supérieur aux mots que les mots le sont aux signes muets des animaux » (p. 71). Ce langage leur sert surtout à se communiquer instantanément des connaissances portant sur le monde extérieur, il se combine ainsi avec la capacité d'action mentale directe sur la matière ${ }^{22}$.

19 Par exemple, lors d'une conversation avec Arlène (14 ans), Jean Arbalaid lui demande comment elle peut être une personne et à la fais une partie intégrante du groupe : «A : Mais je reste moi-même, Jean, je ne pourrais cesser d'être moi-même. J : Les autres sont aussi toi ? A : Oui. Mais je suis eux. J : Mais qui commande à ton corps ? A : Moi, évidemment. J : Ou'arriverait-il si les autres voulaient diriger ton corps à ta place ? A. Pourquoi le voudraient-ils ? $\mathrm{J}$ : Si tu faisais quelque chose qu'ils désapprouvent (...) ? A : Comment le pourrais-je ? (...) Pouvez-vous faire quelque chose que vous désapprouvez? $\mathrm{J}$ : J'ai bien peur que oui, J'en suis fort capable. Et je le fais parfois. A : Mais je ne comprends pas. Pourquoi le faites-vous, alors ? » (p. 70).

20 « Seul le dévouement total de la mère pour son enfant peut te donner une petite idée de l'amour qui les lie les uns aux autres - mais ici, aussi, ils sont différents de nous, cet amour est plus profond encore que l'amour maternel » (p. $69)$.

21 « Lorsque l'un d'eux avait des ennuis - je cite leurs propres paroles - ils l'aidaient à retrouver la paix ; lorsque l'un était malade, tous le soignaient » (p. 69).

22 « Si l'un d'eux regardait quelque chose, il n'avait pas besoin de le dire aux autres, qui le voyaient avec ses yeux. Même pendant leur sommeil, ils rêvaient ensemble » (p. 71). 


\section{L'harmonie des rapports sociaux}

$\underline{\text { Retour à la table des matières }}$

Il est impossible de dire si, au sein du groupe d'enfants et d'adolescents, il y a hiérarchie ou égalitarisme ${ }^{23}$. Si chacun est à la fois lui-

23 S'il est difficile de déceler des rapports de leadership et de pouvoir au sein du groupe d'enfants, il n'en va pas de même en ce qui concerne le groupe d'adultes et l'évolution de ses rapports avec le groupe d'enfants, ni en ce qui concerne les rapports entre les membres du projet Arbalaid et le gouvernement américain. Dans ce cas, par exemple, tout se fonde sur la ruse, la fausse représentation, les manœuvres de retardement. Il n'est jamais question d'un affrontement direct avec l'ancienne société : le projet Arbalaid est financé par l'armée en tant que moyen pour former des surhommes dans une lutte des cerveaux contre l'U.R.S.S., tandis qu'il s'agit en fait de permettre l'éclosion d'une humanité nouvelle, complètement détachée de la guerre et de la haine. À la fin ... /... du récit, au moment où les adolescents disposent de connaissances et de capacités psychiques qui les rendent presqu'invulnérables, il n'est pas question d'un affrontement direct, les mesures prises visant essentiellement à rendre la réserve inaccessible afin de pouvoir y amener de nouveaux bébés et augmenter ainsi le nombre des hommes-plus.

Au sein du groupe d'adultes, par contre, le leadership du couple Arbalaid ressort nettement. Ce sont Mark et Jean qui élaborent l'ensemble du projet et qui dirigent son développement, ce sont eux qui convainquent Harry Felton de les aider et d'enrôler te professeur Goldbaum. Ils obtiennent de plus les fonds nécessaires et procèdent au recrutement des adultes devant participer à l'expérience. Leur leadership n'est pas remis en cause du fait que Harry joue un rôle majeur lors de la réalisation des premières phases du projet, et que le leadership moral gravite de plus en plus autour de Goldbaum à mesure que se déroule l'expérience. Quant au couple lui-même, il semble bien que l'influence de Jean y soit prépondérante : c'est elle qui écrit toutes les lettres et qui est identifiée au projet par les autorités gouvernementales.

Entre les adultes et les enfants, enfin, le rapport d'influence évolue tout au long du récit. Au départ, les adultes exercent une influence presqu'absolue : ils choisissent les enfants, déterminent leur milieu matériel et humain, font le tri des connaissances à leur transmettre, pratiquent une éducation collective. Très vite, toutefois, à mesure que se développent les capacités intellectuelles et psychiques, s'amorce un processus qui renversera complètement la relation de dépendance initiale ; à tel point que, dans sa dernière lettre, Jean écrit : « Je les appelle toujours les enfants alors qu'en réalité nous sommes les enfants et ils 
même et tous les autres, il est difficile d'imaginer l'existence d'un leadership quelconque ou d'une forme même mineure de division spécialisée du travail : ayant accès à la mémoire et à l'expérience de tous, n'importe qui est capable à n'importe quel moment d'accomplir n'importe quelle tâche ${ }^{24}$.

\section{Les caractéristiques utopiques}

$\underline{\text { Retour à la table des matières }}$

Après avoir mis en évidence, dans le récit de Fast, les principaux traits qui le rattachent aux représentations les plus constantes de l'état idéal de perfection des rapports sociaux, il nous reste maintenant à en dégager les caractéristiques proprement utopiques : l'état idéal est en effet situé en ce monde, dans l'avenir, il est réalisable de par le seul effort humain, grâce à la raison scientifique et à la planification des rapports sociaux (politique, stratégie, expérimentation),

\section{Les moyens de réalisation de l'état idéal}

Un examen attentif des diverses phases d'élaboration et de réalisation du projet des Arbalaid permet de dégager la part de la rationalité (scientifique et politique) dans l'avènement des hommes-plus. Si la

sont les adultes » (pp, 53-54). À la fin, avec l'établissement de la barrière temporelle, l'inversion du rapport de dépendance est achevée : ce sont les adolescents qui protègent les adultes.

24 Le texte de Fast donne quelques indications permettant de se faire une idée générale du partage des tâches au sein du groupe d'adultes. Les Arbalaid s'occupent du leadership général, des contacts avec les autorités, du soin et de l'éducation des enfants. Le professeur Goldbaum participe aux rencontres avec les autorités, joue le rôle de principal analyste de ce qui se passe aux étapes cruciales dans le développement du groupe d'enfants. Comme tous les autres, il participe également aux soins généraux (amour, sécurité) des petits. En ce qui concerne l'éducation intellectuelle, enfin, les adultes se répartissent selon plusieurs spécialités: psychologues, une mathématicienne et une sémanticienne, historiens, psychiatres, un physicien et une physicienne. 
part de la science et de la stratégie rationnelle semble bien prépondérante dans le processus d'ensemble, nous verrons qu'un rôle important est également accordé aux facteurs affectifs et aux pouvoirs psychiques.

\section{L'élaboration du projet « hommes-plus »}

Psychologues travaillant depuis plusieurs années pour le Service de Santé Publique du gouvernement des États-Unis, les Arbalaid adoptent la théorie de la prééminence du milieu dans le processus de formation de l'être humain. Ils posent l'hypothèse voulant qu'une modification contrôlée du milieu psychosociologique puisse, dès la petite enfance, favoriser l'éclosion d'une humanité nouvelle. Ils appuient cette hypothèse sur diverses observations concernant les enfants sauvages et les enfants surdoués 25 . Jusqu'ici, la démarche est strictement scientifique. Mais, pour vérifier l'hypothèse, il faut créer un milieu favorable à l'éclosion des hommes-plus, ce qui représente une longue et coûteuse expérimentation, pour la réalisation de laquelle la seule démarche scientifique ne suffit plus : on doit élaborer une stratégie d'ensemble, et surtout obtenir le financement adéquat. Le pivot de toute la stratégie constituera à tromper le gouvernement, auquel on

25 Les observations en question sont présentées sur un mode très objectif. Elles sont de deux types. a) Des observations récentes, toutes authentifiées par des témoins sous serment, sur les enfants sauvages (neuf cas en Inde, au Canada, en Russie, en Afrique et aux États-Unis ; trente-et-un cas plus anciens rapportés par le folklore depuis quatorze siècles). Dans presque tous ces cas, l'enfant est devenu semblable à l'espèce qui l'a recueilli. b) On connaît plusieurs centaines de cas de personnes ayant des enfants d'un 0.1. de 150 et plus. Mais $10 \%$ seulement de ces enfants réussissent dans leur carrière, les autres se suicidant ou sombrant dans une détresse psychique plus ou moins incurable. Un potentiel incroyable est ainsi ramené à l'imbécillité normale, le Q.I. de la plupart des surdoués dégringolant avec l'âge. Les Arbalaid déduisent de tout cela que, si l'homme n'emploie qu'une si petite partie de ses facultés, c'est que le milieu socio-culturel l'empêche d'utiliser le reste : « ... il sont enfermés dans et modelés par leur environnement humain aussi sûrement et inexorablement que ta jeune Indienne l'était par les loups et ton jeune Bantou par les babouins » (cf. pp. 26-28). 
proposera un projet visant officiellement à former des surhommes pour la lutte des cerveaux contre l'U.R.S.S. (pp. 28-29). Et, en obtenant que nul observateur ne mette les pieds dans la réserve avant un temps assez long, on s'assurera du délai nécessaire pour que le véritable projet atteigne un seuil irréversible (pp. 46-52).

\section{La mise en marche}

Dès la mise en marche du projet, il apparaît que les moyens mis en oeuvre dépassent le cadre d'une stricte démarche rationnelle, d'ordre scientifique et politique : amour, affection et humanitarisme y jouent également un rôle important.

Parmi les critères de recrutement des enfants, un fort Q.I. est essentiel. C'est une méthode scientifique qui permet de le déterminer avant même l'apparition du langage (la méthode Goldbaum). D'autres critères interviennent toutefois : choix de diverses races, recrutement dans les pays pauvres et parmi les orphelins de guerre (pp. 32-34). Les adultes participant à l'expérience sont, quant à eux, choisis en fonction de leur capacité de transmettre aux enfants l'essentiel du savoir scientifique de l'humanité et de la volonté de leur assurer collectivement amour et sécurité (pp. 41-43).

Harry Felton, enfin, qui est essentiel à toutes ces phases de l'opération - c'est lui qui retrouve Goldbaum, le convainc d'adhérer au projet et l'aide à trouver les bébés -, travaille pour sa soeur à la fois par désoeuvrement et par affection (pp. 35-40).

\section{La réalisation}

Le résultat global du projet Arbalaid, c'est l'apparition d'une communauté fusionnelle de télépathes, dont les membres possèdent dès l'enfance une capacité foudroyante d'apprentissage et des pouvoirs inouïs d'action de la pensée sur la matière. Dans son évaluation des facteurs qui ont assuré le succès de l'entreprise, Jean Arbalaid accorde 
une grande importance à la transmission des savoirs essentiels de l'humanité, dans un contexte d'amour et de sécurité ; c'est-à-dire à tous les facteurs nouveaux qui ont été introduits de façon planifiée, dès le départ, dans le milieu socio-psychologique des futurs hommes-plus. Mais on ne saurait pour autant conclure à une totale prééminence de la science. Les hommes-plus sont très savants, bien sûr, mais ils sont aussi liés entre eux par un amour très intense et ils sont doués de pouvoirs psychiques échappant à la science stricto sensu. De plus, dans son évaluation, Jean Arbalaid finit par privilégier le rôle de l'amour :

Nous avons pris un groupe d'enfants merveilleusement doués, nous les avons comblés d'amour, nous leur avons offert la sécurité et enseigné la vérité - mais je crois que c'est le facteur affectif qui fut le plus important (p. 54).

Comme moyen de passage à l'état idéal, il est impossible d'affirmer que la démarche rationnelle est seule en cause, de par le jeu de la science et de la stratégie politique impliqué dans le projet. Et cela même si on a parfois l'impression que les "facteurs affectifs" d'amour et de sécurité sont traités comme variable « lourde » dans une expérimentation scientifique...

\section{Le monde extérieur}

Le rapport entre les hommes-plus et l'humanité ordinaire en est un d'incompatibilité. Pour permettre l'émergence des hommes-plus, il a fallu se retirer le plus possible d'un monde bloquant l'apparition des nouvelles capacités ; et, une fois celles-ci développées, leurs porteurs ont dû se préserver de l'extérieur en érigeant une barrière temporelle autour de la réserve : ainsi protégés, les adolescents pourront aller chercher d'autres bébés, les éduquer comme eux, fonder d'autres réserves (pp. 76-79). Ainsi, la défense et l'essentiel essaimage des hommes-plus sont en bonne partie rendus possibles grâce à leurs grandes connaissances de la physique. Mais, là encore, la science ne 
pourrait rien, ni la stratégie, sans des capacités psychiques qui leur échappent.

\section{Les pouvoirs psychiques}

Comme nous l'avons déjà mentionné, ces pouvoirs ne sont pas réductibles à la science. Mais, s'ils la dépassent, ils sont en parfaite continuité avec elle : la télépathie permet aux enfants de se créer un langage qui assure la transmission presqu'instantanée des informatîons et des connaissances, l'action directe de la pensée sur la matière tient compte de ses structures physiques (réarranger les électrons, etc.). De plus, les pouvoirs n'échappent pas à la rationalité scientifique pour tout ce qui concerne l'explication de leur apparition. Lors des premiers signes de la présence d'un fonctionnement télépathique chez les enfants, les savants du groupe adulte y voient une confirmation de leur hypothèse de départ : la télépathie a pu être bloquée depuis très longtemps dans les sociétés humaines par un milieu défavorable, face auquel l'enfant doit très tôt élever des barrières mentales, processus qui atrophie de façon irréversible des secteurs entiers de l'esprit (pp. 6162). C'est d'ailleurs cette explication qui sera plus tard reprise et complétée par les adolescents eux-mêmes :

Ce secteur du cerveau d'où nous viennent nos facultés doit être ouvert avant la puberté, sinon les tissus changent et les cellules cérébrales perdent tout leur potentiel de développement (p. 76).

\section{La conception de l'espace}

\section{$\underline{\text { Retour à la table des matières }}$}

Au début comme à la fin du récit, la réserve où se développe l'humanité nouvelle est un espace clos et défendu contre l'extérieur, quoique de façons différentes et pour des raisons diamétralement opposées. Au début, c'est l'armée américaine qui défend l'accès à la réserve, pour préserver le secret de l'entreprise, ce qui donne aux Arba- 
laid un moyen de contrôler parfaitement le milieu expérimental en en limitant l'accès au maximum ${ }^{26}$. À la fin, l'isolement n'est plus sous le contrôle de l'armée mais sous celui des adolescents : la barrière temporelle leur donne la possibilité de se protéger et d'amorcer l'essaimage d'une humanité nouvelle.

La clôture de l'espace ne le rend ainsi inaccessible que temporairement. Une fois l'expérience pleinement réussie, l'humanité entière passera à l'état idéal (hommes-plus), la fonction de clôture ne représentant du début à la fin qu'un moyen d'assurer l'éclosion et la propagation d'une humanité nouvelle :

Dans notre petite réserve, à l'abri du monde, l'homme devient ce qu'il était destiné à être (P. 66).

\section{La conception du temps}

$\underline{\text { Retour à la table des matières }}$

Malgré certaines déviations et forces de ralentissement ${ }^{27}$, l'histoire de l'humanité se déroule ici dans un temps linéaire et irréversible, positivement valorisé : il y aura dans le proche futur avènement d'une humanité nouvelle :

L'espèce humaine possède en elle les germes d'une nouvelle race. Appellela la race des surhommes ou des hommes-plus, appelle-la comme tu voudras. Il y a longtemps que ces hommes supérieurs existent, des centaines

26 « On nous a attribué un domaine de 320 hectares dans le nord de la Californie. Nous voulons y établir un «milieu » -sous la garde de l'armée. Au début, le monde extérieur sera entièrement banni du domaine. Personne ne pourra venir troubler ce milieu, placé sous contrôle permanent » (p. 32).

27 « Parce que nous admettons que quelque chose a mal tourné à un certain moment dans l'histoire de la civilisation, nous allons remonter aux structures sociologiques de la préhistoire » (p. 41). Même dans une optique aussi résolument utopiste, on n'échappe pas complètement au prestige d'un retour régénérateur aux origines... 
de milliers d'années peut-être ? Mais ils sont enfermés dans et modelés par leur environnement humain (pp. 25-26).

L'expérience qui conduit à une telle émergence est entièrement le fait de l'homme ${ }^{28}$, qui doit toutefois conduire cette tâche avec humilité et tenir compte de la possibilité d'un échec ${ }^{29}$. La réussite du projet sera présentée par Jean Arbalaid comme un événement radicalement nouveau, jamais vu précédemment dans toute l'histoire de l'humanité. On assiste pour la première fois «à la pleine éclosion de l'esprit humain »(p. 58), les enfants de la réserve sont «les premiers enfants normaux que ce monde ait jamais connus » (p. 56). Il s'agit là d'un accomplissement unique et l'humanité commence, avec les hommesplus, « à réaliser sa vraie destinée » (p. 66), « tout ce que nous avons fait ne sera rien comparé à ce que ces enfants pourront apprendre et créer » (p. 65) ; et «l'histoire n'a jamais connu de situation semblable » (p. 62).

Comme on doit s'y attendre après de tels propos, le récit de Jean Arbalaid se termine par un accent de triomphe eschatologique, comme une annonce sécularisée de la Fin des Temps et de l'Avènement du Royaume sur la Terre :

L'homme changera, il deviendra ce pourquoi il a été fait, son amour et son savoir s'étendant à tous les univers des firmaments. La fin des guerres, de la haine, de la faim, de la maladie et de la mort : n'est-ce pas le rêve éternel de l'homme (p. 81)?

28 «... si nous entreprenons de créer l'homme, nous devons le faire en toute humilité, Nous devons être l'outil et non l'artisan, et si nous réussissons, nous serons inférieurs aux résultats de nos efforts » (pp. 39-40).

29 « L'expérience peut échouer, mais, si elle réussit, ce sera l'événement le plus significatif de notre époque » (p. 39). 


\section{Pour conclure}

$\underline{\text { Retour à la table des matières }}$

La pertinence de la typologie présentée en première partie serait évidemment mieux assurée si nous avions pu présenter, sous le modèle de l'analyse précédente, plusieurs cas de mythes paradisiaques, d'utopies et de formes intermédiaires (millénarisme, âge d'or). Comme nous l'avions déjà souligné au départ, il nous a toutefois paru de loin préférable de fournir l'analyse d'un seul cas, assez détaillé pour permettre de saisir les règles d'utilisation de la typologie, plutôt que de justifier celle-ci superficiellement à l'aide de plusieurs illustrations rapides. Cela n'exclut nullement la nécessité de procéder ultérieurement à une analyse comparative de plusieurs cas, cette démarche étant la seule à pouvoir établir si la typologie proposée permet effectivement de rendre compte de la variation des diverses formes de l'état idéal de perfection sociale.

Pour l'instant, nous nous contenterons d'une dernière remarque quant aux règles d'application d'une telle typologie à un matériel donné. En premier lieu, ainsi qu'en fait foi la précédente analyse, la réduction (simplification et résumé) du matériel examiné ne doit pas faire abstraction d'éléments indispensables à sa cohérence interne. Quiconque se donnera la peine (ou le plaisir, pour qui apprécie la sciencefiction) de lire en entier la nouvelle de Fast se rendra compte que cette première règle fut ici respectée. N'importe quel matériel ne peut être classé utilement grâce à la typologie, qui ne concerne que des récits ou des pratiques dont le propos central a trait à l'état idéal de perfection sociale.

En second lieu, l'interprétation du matériel se doit d'être minimale, et la moins contournée possible. Ce qui est explicitement formulé importe seul. Si certains traits s'avèrent ambigus, mieux vaut ne pas les 
classer que les forcer dans un sens ou dans l'autre par un souci déplacé d'exhaustivité. Ainsi, par exemple, nous aurions «interprété » à outrance le texte de Fast si nous avions tenu à montrer que, du point de vue des moyens d'accès à l'état idéal, la raison y emportait tout. Il suffisait qu'elle joue un rôle, central, à côté de l'affectivité, et que ni l'une ni l'autre ne puissent se ramener aux types de moyens d'accès caractéristiques du mythe paradisiaque.

Toute tâche de classement du matériel qu'étudie une discipline donnée est ingrate, en partie arbitraire, souvent à reprendre sur de nouvelles bases. Ce qui n'est toutefois pas une raison valable pour s'en dispenser à l'aide de ces raccourcis théoriques ou empiristes dont les sciences humaines souffrent encore considérablement.

\section{Appendice : Résumé de la nouvelle de Fast}

$\underline{\text { Retour à la table des matières }}$

Le récit se déroule durant la période suivant la Seconde Guerre mondiale. Il se présente sous la forme d'un échange épistolaire, au ton très réaliste, entre Harry Felton, un G.I. démobilisé, et sa sœur Jean (sic) Arbalaid. Avec son mari, Mark, celle-ci s'occupe depuis une vingtaine d'années de psychologie développementale. Elle profite de la démobilisation de son frère pour lui demander d'aller faire enquête sur deux cas récents de découverte d'enfants sauvages, une fillette de 14 ans élevée par des loups, en Inde, et un garçon bantou de 11 ans élevé par des babouins, en Afrique du Sud. L'enquête de Harry le conduit à des résultats sans équivoque : l'enfant éduquée

par les loups se comporte comme une louve, celui éduqué par les babouins se comporte comme un babouin : "L'homme est incapable de devenir un homme par ses seuls moyens ; il est le résultat des autres hommes, de la totalité de l'expérience humaine » (p. 22). 
Ayant reçu les observations de son frère, pressée par les questions de celui-ci, Jean lui expose les conclusions auxquelles son mari et elle sont arrivés après avoir longuement réfléchi sur le phénomène des enfants sauvages et sur celui des enfants surdoués. La théorie se formule simplement : "L'espèce humaine possède en elle les germes d'une nouvelle race » (p. 25). Ces « hommes-plus » existeraient virtuellement depuis près de 100000 ans mais, emprisonnés par leur milieu social, ils ne parviendraient pas à se développer.

\section{Élaboration et mise sur pied du projet Arbalaid « hommes-plus »)}

Ces conclusions conduisent le couple Arbalaid à élaborer un projet grandiose, visant à créer artificiellement un milieu social, une " réserve " apte à favoriser le développement des hommes-plus. Ayant travaillé durant la guerre au Service de la Santé publique du gouvernement américain, le couple Arbalaid réussit à obtenir des fonds considérables pour un projet visant supposément à former des surhommes pour la "guerre des cerveaux » contre l'Union Soviétique. Cette subvention, et le secret militaire absolu, constituent la couverture idéale pour mener à bien le développement des hommes-plus.

Pour le choix des enfants à impliquer très jeunes dans l'entreprise, les Arbalaid ont besoin d'une méthode permettant de déterminer le Q.I. avant l'apparition de la parole. Harry se verra donc confier la mission de retrouver celui qui a élaboré cette méthode, le professeur Hans Goldbaum. Ayant bientôt retrouvé ce dernier à Londres, il lui explique le projet Arbalaid et le convainc d'y participer. Les deux hommes sont alors chargés de trouver une quarantaine de bébés, de Q.I. très élevé, parmi les orphelins de guerre, les enfants de parents pauvres, sur tous les continents. La recherche, l'« achat », le transport et les soins aux enfants sont subventionnés par le gouvernement américain. 
Les enfants sont acheminés vers la réserve, le milieu de l'expérience un domaine de 320 hectares dans le nord de la Californie, d'où toute influence extérieure sera bannie jusqu'à l'adolescence des enfants. Ceux-ci y sont pris en charge par une quarantaine de savants, jouant le rôle de parents collectifs. Ce groupe a pour mission de transmettre les connaissances essentielles acquises par l'humanité, dans une ambiance d'amour et de sécurité.

\section{La réalisation du projet}

Après avoir aidé à acheminer les bébés vers la réserve, Harry Felton retourne à la vie civile et perd tout contact avec le projet jusqu'en 1964. Il reçoit alors la lettre de sa sœur, contenant un document qu'elle lui recommande de ne lire qu'en cas d'extrême nécessité. L'année suivante, Harry est convoqué à la Maison Blanche par le Secrétaire à la Défense, qui lui raconte l'évolution du projet depuis son retour à la vie civile.

Pendant quinze ans, tel que convenu, aucun observateur extérieur ne fut envoyé dans la réserve. Les quelques conférences et rapports du professeur Goldbaum et des Arbalaid n'ont révélé aucun détail précis de ce qui s'y passait. Ces quinze années écoulées, ils obtinrent un nouveau délai de trois ans avant l'envoi de représentants gouvernementaux dans la réserve. Puis, après s'être vu refuser un nouveau délai, ils donnèrent leur accord pour une visite officielle. Mais les représentants de Washington ne trouvèrent à la place de la réserve qu'une étendue froide et grise. Même " une petite bombe atomique » ne put venir à bout de ce néant.

Devant la gravité de la situation qui vient de lui être rapportée par le Secrétaire à la Défense, Felton décide de prendre connaissance avec lui du document de sa soeur. Ce dernier relate en détail le déroulement de l'expérience. Dans un milieu très favorable et totalement coupé du monde extérieur, les enfants assimilent très vite, puis développent, de 
façon prodigieuse, les connaissances transmises par les adultes. C'est ensuite « l'éclosion totale de l'esprit humain », le développement de la télépathie et de la psychokinésie, qui entraînent l'apparition d'une communauté fusionnelle, la disparition de la solitude et de la mort, de la souffrance et de la haine.

Après quinze ans d'un tel développement, les enfants, devenus adolescents, convoquent une réunion de tous les membres de la réserve. Ils s'objectent à toute intrusion des experts gouvernementaux et persuadent les Arbalaid et le professeur Goldbaum d'obtenir un nouveau délai de trois ans, pendant lequel ils parcourront le monde à la recherche de nouveaux bébés, devant être éduqués dans la réserve. Une barrière temporelle est ensuite mise au point, barrière qui, en déplaçant d'un dix-millième de seconde la réserve dans le passé, la rend inaccessible au monde extérieur.

La réaction du Secrétaire à la Défense à la lecture du document est immédiate. Il faudra tout mettre en oeuvre pour franchir la barrière et détruire ces enfants sans Dieu et sans morale, cette menace contre l'humanité, avant que le virus ne se propage : les savants trouveront bien un moyen, ce n'est qu'une question de temps, ils trouvent toujours. 


\section{BILIOGRAPHIE}

$\underline{\text { Retour à la table des matières }}$

BARRET-GURGAND

1981 Le Roi des derniers jours. Paris. Hachette.

BOAS G.

1966 The Cuit of Childhood. Londres : Warburg Institute.

BURRIDGE K.O.L.

1969 New Haven, New Earth : A Study of Millenarian Activities. Oxford-Blackwell.

CAILLOIS R.

1967 «Temps circulaire, temps rectiligne », Obliques. Paris : Fata Morgana.

CLASTRES H.

1975 La Terre sans mal. Paris : Éditions du Seuil.

COHN N.

1967 Warrant for Genocide. New York. Oxford University Press.

1970 The Pursuit of the Millenium. New York : Oxford University Press.

CORBIN H. 
1953 «Terre céleste et corps de résurrection d'après quelques traditions iraniennes », Eranos Jahrbuch, XXII : 97-194.

1963 «Au pays de l'imam caché », Eranos Jahrbuch, XXXII : 3187.

\section{DANIÉLOU J.}

1953 « Terre et paradis chez les Pères de l'Église », Eranos Jahrbuch, XXII : 433-472.

DEBOUT S.

1978 L'Utopie de Charles Fourier. Paris : Payot.

DESANTI D.

1970 Les Socialistes de l'utopie. Paris : Payot.

\section{DESROCHES H.}

1969 Dieux d'hommes. Dictionnaires des messianismes et millénarismes de l'ère chrétienne. Paris : Mouton.

1972 Les dieux rêvés. Théisme et athéisme en utopie. Paris : Desclée.

1973 Sociologie de l'espérance. Paris : Calmann-Lévy.

1975 La Société festive. Paris : Éditions du Seuil.

1978 «Sexe et mariage en utopie », Préface à P.B. Noyes, La Maison de mon père. Paris : Belland.

DUMONT L.

1966 Homo hierarchicus. Paris : Gallimard.

1977 Homo aequalis I. Paris : Gallimard.

1983 Essai sur l'individualisme. Paris : Éditions du Seuil.

DUVEAU G. 
1961 Sociologie de l'utopie. Paris: Presses Universitaires de France.

ELIADE M.

1949 Traité d'histoire des religions. Paris : Payot.

1952 Images et symboles. Paris : Gallimard,

1957 Mythes, rêves et mystères. Paris : Gallimard.

1959 Naissances mystiques. Paris : Gallimard.

1962 Méphistophélès et l'androgyne. Paris : Gallimard.

1963 Aspects du mythe. Paris : Gallimard.

1969 Le Mythe de l'éternel retour. Paris : Gallimard.

1971 La Nostalgie des origines. Paris : Gallimard.

EURICH N.

1967 Science in Utopia. Cambridge : Cambridge University Press.

FAST H.

1962 Au seuil du futur. Verviers : Gérard et Cie.

FERGUSON J.

1975 Utopias of the Classical World. New York : Cornell University Press.

GUHL M.C.

1972 «Les Paradis ou la configuration mythique et archétypale du refuge », Circé, 3 : 11-103.

HOBSBAWM E.J.

1963 Les Primitifs de la révolte dans l'Europe moderne. Paris: Fayard. 


\section{LANTERNARI V.}

1979 Les mouvements religieux des peuples opprimés. Paris : Maspero.

LAPOUGE G.

1978 Utopie et civilisation. Paris : Flammarion.

MANHEIM K.

1956 Idéologie et utopie. Paris : Rivière. [Texte disponible dans Les Classiques des sciences sociales. JMT.]

MANUEL F.E. et F.P. Manuel

1979 Utopian Thought in the Western World. Cambridge : Belknap Press of Harvard University.

MUCCHIELLI R.

1960 Le Mythe de la cité idéale. Paris : Presses Universitaires de France.

MUMFORD L.

1966 The Story of Utopias. New York.

PAZ O.

1976 Point de convergence. Paris : Gallimard.

QUEIROZ M.I. Pereira de 
1968 Réforme et révolution dans les sociétés traditionnelles. Paris : Anthropos.

RACINE L.

1983 "Paradis, âge d'or, royaume millénaire et cité utopique », Diogène, 122 : 130-147.

RIGAUX B.

1932 L'antéchrist et l'opposition au Royaume messianique dans l'Ancien et le Nouveau Testament. Paris.

RUYER R. 1950

L'Utopie et les utopies. Paris : Presses Universitaires de France.

SCHLANGER J.E.

1971 «L'enfance de l'humanité », Diogène, 73 : 41-70.

SERVIER J.

1967 Histoire de l'utopie. Paris : Gallimard.

SUVIN D.

1977 Pour une poétique de la science-fiction. Montréal : Presses de l'Université du Québec.

THRUPP S.L. (éd.)

1962 Millenial Dreams in Action. La Haye : Mouton.

VERSINS P. 
1972 Encyclopédie de l'utopie, de la science-fiction et des voyages extraordinaires. Lausane.

VULLIAUD P.

1952 La Fin du monde. Paris.

WORSLEY P.

1957 The Trumpet Shall Sound. A Study of « Cargo » Cults in Melanesia. Londres.

WUNENBURGER J.J.

1979 L'utopie ou la crise de l'imaginaire. Paris : Delarge.

Fin du texte 